\title{
PREVALÊNCIA DE HELMINTÍASES EM APÊNDICES CECAIS
}

\author{
PREVALENCE OF HELMINTIASIS IN CECALAPPENDICES
}

\author{
Mirian Beatriz Gehlen Ferrari, TCBC-RS ${ }^{1}$ \\ Rubens Rodriguez ${ }^{2}$
}

\begin{abstract}
RESUMO: Objetivo: Relatar a prevalência do diagnóstico de helmintíases em apêndices cecais removidos por procedimentos cirúrgicos em municípios do Estado do Rio Grande do Sul e enviados para o Instituto de Patologia de Passo Fundo. Método: Estudo transversal de prevalência. Analisados 3.646 laudos de apêndices cecais do Instituto de Patologia de Passo Fundo, no período de 01 de maio de 1995 a 24 de abril de 2001. Resultados: A prevalência de helmintos nas peças cirúrgicas foi de 3,07 \% (n=112), com a enterobíase perfazendo 66,07\% ( $\mathrm{n}=74)$ dos casos, seguidos por angiostrongilíase 20,54\% $9(\mathrm{n}=23)$, multiparasitose 5,35\% $(\mathrm{n}=6)$, teníase $4,47 \%(\mathrm{n}=5)$, ascaridíase $2,68 \%(\mathrm{n}=3)$ e um caso de ovo de helminto não identificado. $\mathrm{O}$ diagnóstico histopatológico de apendicite aguda, ocorreu em 75,09\% dos casos associados à helmintíase. Conclusão: A prevalência do diagnóstico histopatológico de helmintíases em apêndices cecais ressecados, por provável diagnóstico clínico de apendicite ou abdômen agudo, encontrados em nosso meio foi semelhante aos descritos mundialmente. $\mathrm{O}$ exame histopatológico pode revelar helmintos ou ovos durante ou após a intervenção.
\end{abstract}

Descritores: Apêndice; Ceco; Apendicites; Helmintos; Doenças parasitárias.

\section{INTRODUÇÃO}

As parasitoses intestinais, ou enteroparasitoses são determinadas por macroparasitas (helmintos) ou microparasitas (vírus, bactérias e protozoários), são de distribuição universal, com maior prevalência e sintomatologia nos países subdesenvolvidos, atingindo 90\%, onde as condições sanitárias são mais deficientes. A gravidade das parasitoses parece estar aumentando mundialmente devido a um aumento da resistência a antimicrobianos e a um número crescente de hospedeiros susceptíveis, sobretudo portadores de HIV ${ }^{1,2}$. No Brasil, com diferenças regionais, a contaminação do ambiente é intensa e a prevalência de parasitoses intestinais é elevada. Observou-se que entre assalariados da cidade de São Paulo, 62,8\% dos indivíduos eram parasitados por helmintos e protozoários, cifra maior que a prevalência mundial de $25 \%{ }^{2}$.
A sintomatologia das helmintíases apresenta ampla variabilidade: da ausência de manifestação clínica - a grande maioria - a raríssimos casos graves e letais, entre eles as complicações cirúrgicas abdominais. Como a clínica é inespecífica, para o diagnóstico das helmintíases intestinais é preconizado basicamente o exame protoparasitológico: exame parasitológico de fezes (EPF), com análise de três amostras de fezes; eventualmente podem ser utilizados: tamização, anal swab, intradermorreação, biópsias e, raramente, o diagnóstico é realizado durante procedimentos cirúrgicos com visualização ou palpação de parasitas ou de suas complicações e pelo exame histopatológico da peças cirúrgicas ${ }^{3-6}$.

Entre as complicações cirúrgicas intestinais causadas por estes vermes, pode-se citar: apendicite aguda, oclusão intestinal, enterite, necrose, perfuração e peritonite. Migrando para outros órgãos abdo-

1. Cirurgiã Geral e Preceptora da Residência Médica em Cirurgia Geral do Hospital da Cidade de Passo Fundo; Mestre em Clínica Médica - Mestrado Insterinstitucional - Universidade Federal do Rio Grande do Sul e Universidade de Passo Fundo.

2. Mestre em Anatomia Patológica; Patologista Cirúrgico do Instituto de Patologia de Passo Fundo; Professor de Patologia da Faculdade de Medicina da Universidade de Passo Fundo.

Recebido em 21/01/2002

Aceito para publicação em 10/02/2004

Trabalho realizado no Instituto de Patologia de Passo Fundo, Passo Fundo - RS. 
minais podem desencadear abscessos hepáticos, colangite, colecistite, pancreatite, infecções ginecológicas e urinárias. $\mathrm{O}$ diagnostico de verminose pode ser realizado no tratamento destas complicações, como no quadro de apendicite aguda ${ }^{4}$.

O presente estudo relata a prevalência do diagnóstico de helmintíases em apêndices cecais removidos, em nosso meio, por procedimentos cirúrgicos, acompanhados ou não de segmentos intestinais, enviados para o Instituto de Patologia de Passo Fundo.

\section{MÉTODO}

Foram analisados todos os laudos do Instituto de Patologia de Passo Fundo (IPPF), no período de 01 de maio de 1995 a 24 de abril de 2001, que na(s) peça(s) cirúrgica(s) que tivesse incluso o apêndice cecal.

O IPPF recebeu peças cirúrgicas que continham o apêndice cecal enviados por 213 médicos de 56 municípios do Estado do Rio Grande do Sul. Encontraram-se helmintos em peças enviadas de 23 destes municípios.

Os critérios de inclusão no estudo foram todos os laudos com diagnóstico de apendicite ou hiperplasia linfóide ou simplesmente parasitose na peça cirúrgica em que o apêndice estava envolvido. Foram considerados exames positivos para parasitoses aqueles que se encontrasse ovos, larvas ou vermes adultos (Figuras 1 e 2). No caso da angiostrongilíase abdominal, também foram incluídos os casos com aspectos morfológicos compatíveis com essa doença ${ }^{3}$. Excluíram-se laudos com diagnóstico de tumor, doenças inflamatórias crônicas in-

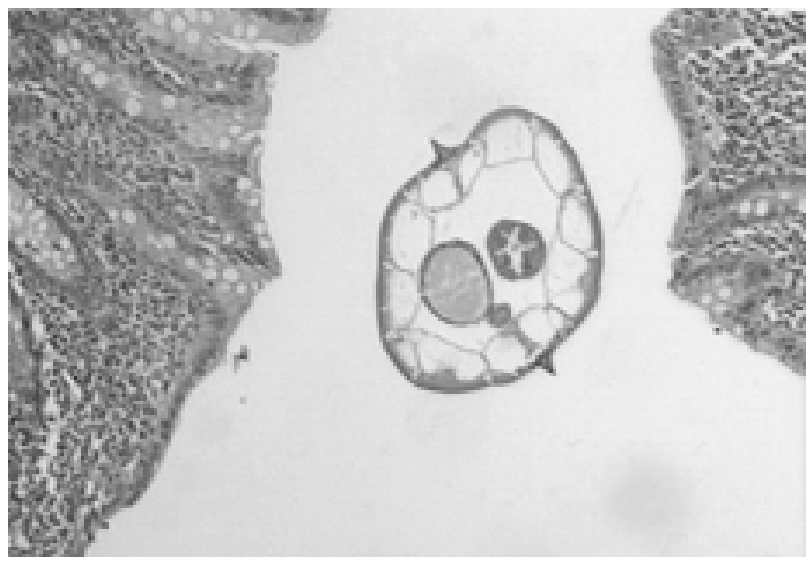

Figura 1 - Apêndice cecal apresentando corte transversal de verme adulto de Enterobius vermiculares. testinais e patologias ginecológicas em que o apêndice estava incluído.

Dos 4.122 laudos, 3.646 preencheram os critérios de inclusão para o estudo e foram analisados, estratificados por faixa etária, pelo diagnóstico histológico da helmintíase e da patologia do apêndice. Relativamente à faixa etária, considerou-se criança até os doze anos incompletos, adolescentes dos doze aos 21 anos incompletos, e adultos, acima de 21 anos de idade, conforme o Estatuto da Criança e do Adolescente. O estudo estatístico foi realizado utilizandose dados descritivos (médias e desvio padrão) e para análise entre grupos utilizou-se o qui-quadrado, considerando significativo alfa de $5 \%$.

\section{RESULTADOS}

Entre os 3.646 laudos selecionados, 56,53\% eram de pacientes do sexo masculino e $43,47 \%$ de pacientes do sexo feminino, com média de idade de $24 \pm 15$ anos (média e desvio padrão).

A prevalência de helmintos nas peças cirúrgicas foi de 3,07 \% ( $\mathrm{n}=112)$, assim distribuídos: enterobíase $66,07 \%$, angiostrongilíase abdominal $20,54 \%$, multiparasitose $5,35 \%$, teníase $4,47 \%$, ascaridíase $2,68 \%$ e ovo não identificado $0,89 \%$. O diagnóstico histopatológico de apendicite, associado à helmintíase, ocorreu em 77,76\% dos casos - 75,09 como aguda; $1,78 \%$ como crônica e $0,89 \%$ eosinofílica - seguido por hiperplasia linfóide em $16,96 \%$ e somente $4,47 \%$ das helmintíases nos apêndices estavam associados a quadros de hiperemia, hemorragia, congestão apendicular ou sem outra alteração histológica específica (Tabela 1).

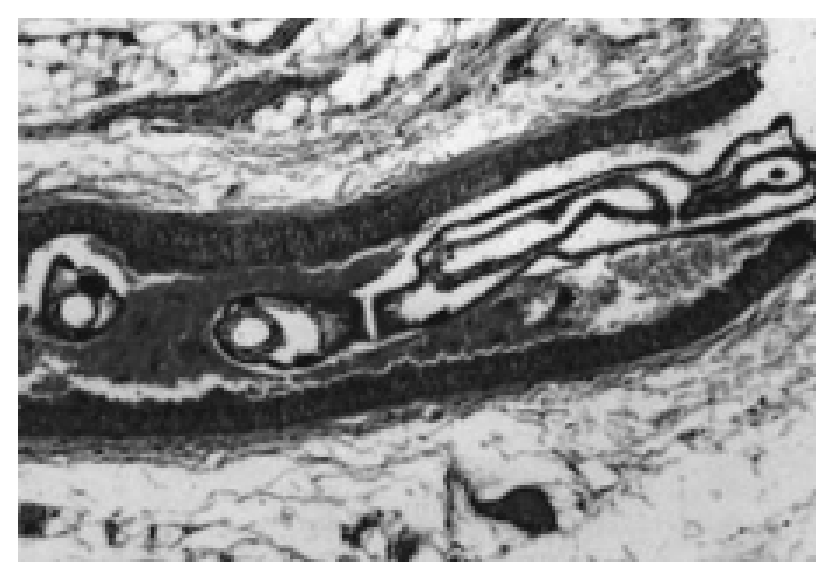

Figura 2 - Artéria de mesoapêndice exibindo verme adulto de Angiostrongylus costaricensis na luz. 
Tabela 1 - Prevalência de diagnóstico histopatológico de helmintíases em apêndices cecais.

Apendicite

\begin{tabular}{|c|c|c|c|c|c|c|c|c|c|c|}
\hline \multirow[b]{2}{*}{ Helmintíase } & \multicolumn{2}{|c|}{$\overline{\text { Aguda }}$} & \multicolumn{2}{|c|}{ Crônica } & \multicolumn{2}{|c|}{ Eosinofílica } & \multicolumn{2}{|c|}{ Outros* } & \multicolumn{2}{|c|}{ Total } \\
\hline & $\mathbf{n}$ & $(\%)$ & $\mathbf{n}$ & $(\%)$ & n & $(\%)$ & $\mathbf{n}$ & $(\%)$ & $\mathbf{n}$ & $\%$ \\
\hline Ascaridíase & 3 & $(2,68)$ & - & - & - & - & - & - & 3 & $(2,68)$ \\
\hline $\begin{array}{l}\text { Angiostrongilíase } \\
\text { abdominal } \\
\text { confirmada }\end{array}$ & 4 & $(3,57)$ & - & - & - & - & - & & 4 & $(3,57)$ \\
\hline $\begin{array}{l}\text { Compatível com } \\
\text { angiostrongilíase } \\
\text { abdominal }\end{array}$ & 16 & $(14,29)$ & - & - & - & - & 3 & $(2,68)$ & 19 & $(16,97)$ \\
\hline Enterobíase & 51 & $(45,54)$ & 1 & $(0,89)$ & 1 & $(0,89)$ & 2 & $(1,79)$ & 74 & $(66,07)$ \\
\hline $\begin{array}{l}\text { Enterobíase e } \\
\text { Ascaridíase }\end{array}$ & 2 & $(1,78)$ & - & - & - & - & - & - & 2 & $(1,78)$ \\
\hline $\begin{array}{l}\text { Enterobíase e } \\
\text { compatível com } \\
\text { angiostrongilíase } \\
\text { abdominal }\end{array}$ & 3 & $(2,68)$ & - & - & - & - & - & - & 3 & $(2,68)$ \\
\hline $\begin{array}{l}\text { Enterobíase e } \\
\text { Estrongiloidíase }\end{array}$ & 1 & $(0,89)$ & - & - & - & - & - & - & 1 & $(\mathbf{0 , 8 9})$ \\
\hline Teníase & 5 & $(4,47)$ & - & - & - & - & - & - & 5 & $(4,47)$ \\
\hline $\begin{array}{l}\text { Ovos de helmintos } \\
\text { não identificados }\end{array}$ & - & - & 1 & $(0,89)$ & - & - & - & - & 1 & $(\mathbf{0 , 8 9})$ \\
\hline Total & 85 & $(75,90)$ & 2 & $(1,78)$ & 1 & $(0,89)$ & 5 & $(4,47)$ & 112 & $(100,00)$ \\
\hline
\end{tabular}

* Refere-se a apêndices com congestão, hiperemia, hemorragia ou sem diagnóstico específico.

A média de idade dos casos de helmintíases foi de $19 \pm 12$ anos (média e desvio padrão), excluindo-se sete casos em que não havia esta informação, com diferença estatisticamente significativa $(\mathrm{p}=0,01)$ para os tipos de helmintos (Tabela 2). Reforçou-se esta diferença $(\mathrm{p}=0,001)$ na análise da faixa etária (Tabela 3): a teníase ocorreu exclusivamente em adultos $(100 \%)$, a ascaridíase em adolescentes $(100 \%)$ e a enterobíase foi maior em crianças $(48,64 \%)$. A multiparasitose foi observada em $40 \%$ dos adultos e crianças e $20 \%$ dos adolescentes. Com relação ao sexo, as diferenças, não foram significativas.

\section{DISCUSSÃO}

Como as verminoses são de distribuição universal, existem incontáveis trabalhos publicados so- bre as complicações abdominais potencialmente cirúrgicas das mesmas. Ochoa ${ }^{4}$, na Colômbia, descreveu complicações abdominais em 311 crianças parasitadas por E. Hystoliticae e por Ascaris lumbricóides: com 145 casos de complicações da ascaridíase incluindo 107 oclusões intestinais, 10 perfurações do apêndice e 28 migrações de ascaris para a árvore biliar ou cavidade peritoneal. Listorto et $a l^{5}$, na Itália, apresentaram 14 casos de enterobíase de apêndice em 1093 apendicectomias. Teles e Fontes ${ }^{6}$ alertaram para os casos de angiostrongylose intestinal com ainda poucos casos no Brasil diagnosticados nas intervenções cirúrgicas. Mukhopadhyay et $\mathrm{al}^{7}$, na Índia, relataram cinco mortes por complicações pósoperatórias, em 104 crianças operadas com oclusão intestinal por ascaridíase. Saxena $e t a l^{8}$, na Alemanha, observaram Enterobius vermiculares na cavi- 
Tabela 2 - Média de idade dos pacientes com diagnóstico histopatológico de helmintíases.

\begin{tabular}{lccc}
\hline Helmintíases (n)* & $\begin{array}{c}\text { Média de idade } \pm \\
\text { desvio padrão (em anos) }\end{array}$ & $\begin{array}{c}\text { Idade Mínima } \\
(\mathbf{e m} \text { anos) }\end{array}$ & $\begin{array}{c}\text { Idade Máxima } \\
(\mathbf{e m ~ a n o s )}\end{array}$ \\
\hline Angiostrongilíase Abdominal (22) & $26 \pm 12$ & 2 & 49 \\
Ascaridíase (2) & $13 \pm 2$ & 11 & 15 \\
Enterobíase (70) & $16 \pm 11$ & 11 & 46 \\
Teníase (5) & $38 \pm 16$ & 23 & 58 \\
Multiparasitas (5) & $18 \pm 7$ & 10 & 39 \\
Ovos não identificados (1) & $5 \pm 0$ & 5 & 5 \\
\hline Total & $\mathbf{1 9 \pm 1 2}$ & $\mathbf{2}$ & $\mathbf{5 8}$ \\
\hline
\end{tabular}

* Em sete laudos não havia a descrição da idade do paciente.

dade abdominal de três pacientes pediátricos submetidos a apendicectomia laparoscópica, logo após a ligadura e ressecção do coto apendicular, corroborando Schnell et al $l^{9}$ (1992) que descreveram um caso de salpingite aguda e crônica em uma paciente com história de apendicite com enterobíase cinco anos antes. Rodriguez et $a l^{10}$ descreveram no Rio Grande do Sul casos de angiostrongilíase abdominal como causa de apendicite aguda em crianças.
Neste estudo, foram selecionadas apenas as helmintíases que envolveram o apêndice cecal: ancilostomíase, ascaridíase, teníase, esquistossomose, angiostrongilíase, estrongiloidíase e enterobíase, e os resultados foram semelhantes aos descritos em outros estudos ${ }^{11-19}$.

A prevalência de diagnóstico histopatológico de helmintíases em apêndices cecais ressecados, por provável diagnóstico clínico de apendicite ou abdôme

Tabela 3 - Distribuição percentual das helmintíases por faixa etária.

\begin{tabular}{|c|c|c|c|c|c|c|c|c|}
\hline \multirow[t]{2}{*}{ Helmintíases* } & \multicolumn{2}{|c|}{$0 \longmapsto 12$ anos } & \multicolumn{2}{|c|}{$12 \longmapsto 21$ anos } & \multicolumn{2}{|c|}{$>$ de 21 anos } & \multicolumn{2}{|c|}{ Total } \\
\hline & n & $(\%)$ & n & $(\%)$ & $\mathbf{n}$ & $(\%)$ & $\mathbf{n}$ & $(\%)$ \\
\hline $\begin{array}{l}\text { Angiostrongilíase } \\
\text { abdominal }\end{array}$ & 3 & $(13,64)$ & 3 & $(13,64)$ & 16 & $(72,72)$ & 22 & (100) \\
\hline Ascaridíase & - & - & 2 & (100) & - & - & 2 & (100) \\
\hline Enterobíase & 34 & $(48,64)$ & 18 & $(25,62)$ & 18 & $(25,62)$ & 70 & (100) \\
\hline Teníase & - & - & - & - & 5 & (100) & 5 & (100) \\
\hline Multiparasitas & 2 & $(40)$ & 1 & (20) & 2 & (40) & 5 & (100) \\
\hline $\begin{array}{l}\text { Ovos não } \\
\text { identificados }\end{array}$ & 1 & (100) & - & - & - & - & 1 & (100) \\
\hline Total Geral & 40 & $(38,09)$ & 24 & $(22,86)$ & 41 & $(39,05)$ & 105 & (100) \\
\hline
\end{tabular}

* Em sete laudos não havia a descrição da idade do paciente. 
agudo, encontrados em nosso meio foi de 3,07\%, semelhante aos descritos mundialmente. $\mathrm{Ojo}^{20}$, na Nigéria, descreveu uma prevalência de 5,4\% em 319 espécimes cirúrgicos apendiculares enviados a histopatologia. Dahlstron $^{21}$, naAustrália, identificou 3,4\% de enterobíase em 1867 apêndices cecais encaminhados para o laboratório de Patologia do Woden Vale Hospital, entre os anos de 1984 e 1988. No presente estudo, diferente da literatura, não houve casos de esquistossomose.
Conclui-se que a prevalência de helmintos intestinais em apêndices cecais - associada ao quadro clinico de apendicite ou de abdômen agudo cirúrgico - nesta amostra foi de 3,07\%, semelhante à mundial. A observação macroscópica da peça cirúrgica, a revisão minuciosa da cavidade abdominal e o exame histopatológico podem revelar helmintos ou ovos durante ou após a intervenção.

\begin{abstract}
Background: The purpose of this study is to report the prevalence of the helmintiases in cecal appendices surgically removed. Method: Transversal study of prevalence. Analyzed 3.646 reports of cecal appendices from the Instituto de Pathologia de Passo Fundo, from May 01, 1995 to April 24, 2001. Results: The prevalence of helmints on the surgical speciments was $3.07 \%(n=112)$, with the enterobiases present in $66.07 \%(n=74)$ of the cases, followed by angiostrongyliasis in $20.54 \%(n=23)$, multiparasitosis $5.35 \%$ $(n=6)$, teniasis $4.47 \%(n=5)$, ascariadiasis $2.68 \%(n=3)$ and one case of an unidentified worm's egg. Males were $53.57 \%$ and $46.43 \%$ were females, with mean age of $19 \pm 12$ years. The histopatological diagnosis of acute appendicitis, occurred in $75.09 \%$ of the cases associated to helmintíasis. Conclusions: It was concluded that helmintíasis histopatological prevalence, in cecal appendices, for possible clinical diagnosis of appendicitis or acute abdome, were similar to the ones described worldwide. The histopatological study can discover helmints or eggs during or after the intervention.
\end{abstract}

Key Words: Cecal; Appendix; Appendicitis; Helminths; Parasitic diseases.

\section{REFERÊNCIAS}

1. Azevedo MF - "Parasitoses causadas por protozoários e helmintos". In Caria CF, Lee HH, Woeltje KF. Trad. Azevedo MF, Hennenmann TLA - Washington Manual de Terapêutica Clínica. $29^{\mathrm{a}}$ Edição. Rio de Janeiro. Guanabara Koogan, 1999, pp. 603-611.

2. Chehter L, Cabeça M - "Parasitoses Intestinais". In Prado FC, Ramos J, Valle JR - Atualização terapêutica: manual prático de diagnóstico e tratamento. $19^{\mathrm{a}}$ edição. São Paulo. Artes Médicas, 1999. pp 279-283.

3. Graeff-Teixeira C, Camillo-Coura L, Lenzi HL Histopathological for criteria the diagnosis of abdominal angiostrongyliosis. Parasitol Res, 1991, 77(7):606611.

4. Ochoa B - Surgical complications of ascariasis. World J Surg. 1991, 15(2):222-227.

5. Listorto G, Ferranti F, Mancini G, et al. - Il ruolo dell'Enterobius vermicularis nell'etiopatogenesi dell' appendicite. Minerva Chir, 1996, 51(5):293296.
6. Telles HMS, Fontes LR - Escargot: mais um perigo a mesa. Nova ameaça a saúde pública e a agricultura. Vetores e pragas, 1998, 1(1):4-8.

7. Mukhopadhyay B, Saha S, Maiti S, et al. - Clinical appraisal of Ascaris lumbricoides, with special reference to surgical complications. Pediatr Surg Int, 2001, 17(5-6):403-405.

8. Saxena AK, Springer A, Tsokas J, et al. - Laparoscopic appendectomy in children with Enterobius vermicularis. Surg Laparosc Endosc Percutan Tech. 2001, 11(4):284-286.

9. Schnell VL, Yandell R, Van Zandt S, et al. - Enterobius vermiculares salpingits: a distant episode from precipitating appendicitis. Obstet Gynecol, 1992, 80:(3 pt 2):553-555.

10. Rodriguez R, Agostini AA, Dias EP. Angiostrongilose abdominal como causa de apendicite aguda em crianças. XXII Congresso Brasileiro de Patologia - Anais. Curitiba-PR. 1999.

11. Lampertico P-Considerazioni clinico-patologiche su 9.952 appendici asportate presso l'Ospedale di Busto Arsizio dal 1966 al 1989. Pathologica, 1990, 82(1082):719-732. 
12. Wiebe BM - Appendicitis and Enterobius vermiculares. Scand J Gastroenterol, 1991, 26(3):336-338.

13. Rahmann H, Pandey S, Mishra PC, et al. - Surgical manifestations of ascariasis in childhood. J Indian Med Assoc, 1992, 90(2):37-39.

14. Ross E, Ruiz ME - La patologia del apendice cecal en nuestro medio. Un analisis de 436 especimenes quirurgicos de apendicectomia. GEN, 1995, 49(2):140-144.

15. Weber G, Borer A, Zirkin HJ, et al. - Schistosomiasis presenting as acute appendicitis in a traveler. $\mathrm{J}$ Travel Med. 1998, 5(3):147-148.

16. Capaldi M, Roncolini G, Calabrese P, et al. - A proposito di ossiuriasi appendicolare: presentazione di un caso e revisione della letteratura. Chir Ital, 2000, 52(1):87-89.

17. Khan GM, Grillo IA, Abu-Eshy SA, et al. - Pathology of the appendix. J Natl Med Assoc, 2000, 92(11):533-535.

18. Mohamed AE, Ghandour ZM, Al-Karawi MA, et al. Gastrointestinal parasites presentations and histological diagnosis from endoscopic biopsies and surgical specimens. Saudi Med J, 2000, 21(7):629-634.
19. Zoguéreh DD, Lemaître X, Ikoli JF, et al. - Les appendicites aiguës au Centre National Hospitalier Universitaire de Bangui, Centrafrique: aspects épidémiologiques, cliniques, paracliniques et thérapeutiques. Sante, 2001, 11(2):117-125.

20. Ojo OS, Udeh SC, Odesanmi WO - Review of the histopathological findings in appendices removed for acute appendicitis in Nigerians. J R Coll Surg Edinb, 1991, 36(4):245-248.

21. Dahlstrom JE, Macarthur EB - Enterobius vermicularis: a possible cause of symptoms resembling appendicitis. Aust NZ J Surg, 1994, 64(10):692-694.

Endereço para correspondência:

Rua Teixeira Soares, 879 / sala 301

Centro

99010-081- Passo Fundo - RS 